\title{
Relationship between serum albumin and pulse wave velocity in patients on continuous ambulatory peritoneal dialysis
}

\author{
Li-Tao Cheng', \\ Li-Jun Tangl,2, \\ Hui-Min Chen ${ }^{1,3}$, \\ Wen Tang', \\ Tao Wang' \\ 'Division of Nephrology, Peking \\ University Third Hospital, Beijing, \\ China; ${ }^{2}$ Division of Nephrology, Qilu \\ Hospital of Shandong University, \\ Ji'nan, China; ${ }^{3}$ Division of Cardiology, \\ Guangdong Provincial Hospital \\ of Traditional Chinese Medicine, \\ Guangzhou, China
}

\begin{abstract}
Background: Hypoalbuminemia is a risk factor for cardiovascular events and mortality in dialysis patients, but the underlying mechanism remains unclear. Meanwhile, increased pulse wave velocity (PWV), the marker of arterial stiffness, has been proved to be an independent predictor of cardiovascular disease. The relationship between serum albumin and PWV in continuous ambulatory peritoneal dialysis patients (CAPD) was studied.

Methods: Sixty-two CAPD patients were studied. The average age was $63 \pm 12$ years and dialysis duration was $23 \pm 22$ months. Serum albumin, C-reactive protein (CRP), and carotidfemoral PWV were measured.

Results: Among these patients, $43.5 \%$ were men. The mean serum albumin concentration was $37 \pm 4 \mathrm{~g} / \mathrm{L}$ and PWV was $11.9 \pm 2.3 \mathrm{~m} / \mathrm{s}$. PWV positively correlated with age $(\mathrm{r}=0.35$, $\mathrm{P}<0.01$ ), diabetes (yes $=1$, no $=0 ; \mathrm{r}=0.292$, $\mathrm{P}<0.05$ ), systolic blood pressure (SBP; $\mathrm{r}=0.493, \mathrm{P}<0.001)$ and $\mathrm{CRP}(\mathrm{r}=0.295, \mathrm{P}<0.05)$, but negatively correlated with serum albumin $(r=-0.357, P<0.01)$. In multiple regression analysis, $\operatorname{SBP}(\beta=0.615, P<0.001)$, age $(\beta=0.414, \mathrm{P}<0.01)$, albumin $(\beta=-0.315, \mathrm{P}<0.05)$ and total cholesterol $(\beta=0.275$, $\mathrm{P}<0.05$ ) were independent determinants of PWV. In a non-inflamed subgroup (CRP $<3 \mathrm{mg} / \mathrm{L}$, $\mathrm{n}=30)$, albumin still negatively correlated with PWV $(\mathrm{r}=-0.66, \mathrm{P}<0.001)$.

Conclusion: Serum albumin inversely correlated with increased PWV in CAPD patients, suggesting that increased arterial stiffness might be the link between hypoalbuminemia and increased cardiovascular mortality in dialysis patients.
\end{abstract}

Keywords: hypoalbuminemia, cardiovascular events, pulse wave velocity, arterial stiffness, peritoneal dialysis

\section{Introduction}

In dialysis patients, a number of studies have suggested that hypoalbuminemia is an independent predictor of increased cardiovascular events and mortality (Foley et al 1996; Wong et al 2002; Cooper et al 2004; Kalantar-Zadeh et al 2005). There are also reports that hypoalbuminemia is closely associated with left ventricular hypertrophy (LVH) in dialysis patients (Foley et al 1995; Enia et al 2001). However, the underlying mechanism by which hypoalbuminemia leads to LVH and increased cardiovascular events remains unclear. In recent years, many studies have also proved that increased aortic stiffness is a risk factor for cardiovascular events and mortality (Benetos et al 1998; Asmar et al 2001; Laurent et al 2001; Hansen et al 2006). It is thus reasonable to hypothesize that there might be a close association between hypoalbuminemia and markers of aortic stiffness.

In our previous study, we found a negative correlation between serum albumin and pulse pressure (PP), the surrogate of arterial stiffness (Vlachopoulos and O’Rourke 2000; Dart and Kingwell 2001; Van Bortel et al 2001) in a group of 
continuous ambulatory peritoneal dialysis (CAPD) patients (unpublished data). However, PP is not the true marker of arterial stiffness. Therefore, in the present study, we employed pulse wave velocity (PWV), the direct measure of arterial stiffness (O'Rourke et al 2002), and explored the relationship between serum albumin and PWV in CAPD patients. To clarify the confounding effect of inflammation, we additionally analyzed the relationship between serum albumin and PWV in a subgroup of patients without evidence of inflammation.

\section{Patients and methods Study population}

In this cross-sectional study, we included all stable patients treated with CAPD in the Peritoneal Dialysis Center of Peking University Third Hospital in May, 2006. The exclusion criteria were: 1) dialysis duration less than 3 months; 2) presence of congestive heart failure; and 3) hypotension (defined as systolic blood pressure/diastolic blood pressure $<90 / 60 \mathrm{mmHg}$ ). Based on these criteria, 62 patients were considered suitable for this study. The study protocol was approved by the ethic committee of our hospital and informed consent was obtained from every patient.

\section{Measurement of carotid-femoral pulse wave velocity}

Aortic PWV was determined using an automatic device, the Complior (Colson, Garges les Gonesses, France) (Asmar et al 1995), which allowed online pulse wave recording and automatic calculation of PWV. Common carotid artery and femoral artery pressure wave forms were recorded noninvasively using a TY-306 Fukuda pressure sensitive transducer (Fukuda, Tokyo, Japan). Measurement was repeated over 10 different cardiac cycles, and the mean value was used for the final analysis. The distance traveled by the pulse wave was measured over the body surface as the distance between the two recording sites (D), while pulse transit time (t) was automatically determined by the Complior. Pulse wave velocity was automatically calculated as $P W V=D / t$. Details, as well as validation of this automatic method and its reproducibility, have been reported previously (Asmar et al 1995). All the PWV measurements were performed by one doctor (L-J Tang) and the intra-observer CV was about $1.74 \%-7.95 \%$.

\section{Measurement of blood pressure}

To ensure the accuracy of measurements, a dedicated renal nurse was in charge of all blood pressure (BP) measurements, and the mercury sphygmomanometer used was calibrated regularly. All measurements were performed in a quiet room. Brachial BP was measured twice in a sitting position after patients had rested more than ten minutes. Systolic blood pressure (SBP) and diastolic blood pressure (DBP) were averaged from values of the two measurements.

\section{Biochemical measurements}

Serum albumin was determined by the bromcresol green method. High sensitive C-reactive protein (CRP) was measured as inflammatory index and a CRP value $\geq 3 \mathrm{mg} / \mathrm{L}$ was considered as the presence of inflammation. This method could not detect CRP levels $<0.01 \mathrm{mg} / \mathrm{L}$. Other biochemical indices, such as serum calcium, phosphate, triglycerides, total cholesterol lipoprotein, low-density lipoprotein, and high-density lipoprotein were determined with standard methods.

\section{Measurements of fluid status}

Extracellularwater(ECW) was measured by multiple-frequency bioelectrical impedance analysis (Xitron Technologies, San Diego, CA). The procedure to perform this measurement was described in detail elsewhere (Cheng et al 2005). Briefly, after patients taking supine position for at least 10 minutes, the standard tetrapolar electrodes were placed on the dorsum of wrist and anterior aspect of the ankle on the left side of the body. Three consecutive measurements were performed over a 2-min period, and ECW was calculated as the mean values of the three measurements.

\section{Statistical analysis}

Continuous variables were expressed as mean $\pm \mathrm{SD}$ while categorical variables were expressed as percentage or ratio. Pearson's and Spearman's correlations were performed to explore the relationships between serum albumin and other variables as appropriate. Multiple regression analysis was performed to determine the relationship between serum albumin and PWV to control the influence of other variables. All tests were two-sided. A value of $\mathrm{P}<0.05$ was taken as statistically significant. All analysis was completed with SPSS software, version 11.0 (SPSS, Chicago, IL).

\section{Results}

\section{Demographic characteristics of the study population}

The demographic characteristics of the patients included in this study were shown in Table 1 . There were 27 male and 35 female patients, with an average age of $63 \pm 12$ years 
Table I Demographic characteristics of the study population

\begin{tabular}{ll}
\hline Number of patients & 62 \\
Sex, male/female & $27 / 35$ \\
Age, year & $63 \pm 12$ \\
Height, cm & $157 \pm 14$ \\
Weight, kg & $61 \pm 17$ \\
Dialysis duration, month & $23 \pm 22$ \\
Antihypertensive medication, Yes/No & $52 / 10$ \\
Primary diagnosis & \\
$\quad$ Chronic glomerulonephritis, & $30.6 \%$ \\
Hypertension & $24.2 \%$ \\
$\quad$ Diabetes & $17.7 \%$ \\
Tubulointerstitial nephritis & $11.3 \%$ \\
$\quad$ Unknown & $16.1 \%$ \\
Systolic blood pressure, $\mathrm{mmHg}$ & $149 \pm 23$ \\
Diastolic blood pressure, $\mathrm{mmHg}$ & $84 \pm 13$ \\
Extracellular water, L & $14.7 \pm 3.2$ \\
Serum calcium, mmol/L & $2.25 \pm 0.23$ \\
Serum phosphate, $\mathrm{mmol} / \mathrm{L}$ & $1.56 \pm 0.43$ \\
Total cholesterol, mmol/L & $4.96 \pm 1.06$ \\
Serum albumin, g/L & $37 \pm 4$ \\
Serum CRP, mg/L & $6.6 \pm 6.8$ \\
rKt/V & $0.48 \pm 0.55$ \\
pKt/V & $1.27 \pm 0.44$ \\
tKt/V & $1.75 \pm 0.55$ \\
PWV, m/s & $11.96 \pm 2.27$ \\
\hline
\end{tabular}

Abbreviations: CRP, C-reactive protein; $\mathrm{rKt} / \mathrm{V}$, renal $\mathrm{Kt} / \mathrm{V} ; \mathrm{pKt} / \mathrm{V}$, peritoneal $\mathrm{Kt} / \mathrm{V}$; $\mathrm{tKt} / \mathrm{V}$, total $\mathrm{Kt} / \mathrm{V}(\mathrm{rKt} / \mathrm{V}+\mathrm{pKt} / \mathrm{V})$; $\mathrm{PWV}$, pulse wave velocity.

old (range 23 to 85 ). The mean dialysis duration in these patients was $23 \pm 22$ months. The mean serum albumin was $37 \pm 4 \mathrm{~g} / \mathrm{L}$. The mean PWV was $11.96 \pm 2.27 \mathrm{~m} / \mathrm{s}$.

\section{Univariate correlation multiple regression analysis}

Univariate correlation analysis between PWV and other possible affecting factors were shown in Table 2. PWV was positively correlated with age $(\mathrm{r}=0.35, \mathrm{P}<0.01)$, diabetic status (yes $=1$, no $=0 ; \mathrm{r}=0.292, \mathrm{P}<0.05$ ), SBP $(r=0.493, P<0.001)$ and CRP $(r=0.295, P<0.05)$, but negatively correlated with serum albumin $(\mathrm{r}=-0.357$, $\mathrm{P}<0.01$; Figure 1A). The correlations between PWV and ECW and phosphate were just borderline $(\mathrm{r}=0.241$, $\mathrm{P}=0.077$ and $\mathrm{r}=-0.244, \mathrm{P}=0.056$, respectively). No correlation was found between PWV and sex (female $=1$, male $=0$ ), height, dialysis duration, DBP, antihypertensive medication (yes $=1$, no $=0$ ), serum calcium, and total cholesterol.

Multiple stepwise regression analysis to determine the relationship between PWV and other possible affecting factors was shown in Table 3. In this model, PWV was treated as dependent variable while a series of factors were treated as independent variables, such as age, sex (female $=1$, male $=0$ ), dialysis duration, diabetic status (yes $=1$, no $=0$ ), SBP, DBP, antihypertensive medication (yes $=1$, no $=0$ ), ECW, serum calcium, serum phosphate, total cholesterol, CRP, and serum albumin. The result of multiple stepwise regression analysis showed that SBP (0.615, $\mathrm{P}<0.001)$, age $(0.414, \mathrm{P}<0.01)$, serum albumin $(-0.315, \mathrm{P}<0.05)$ and total cholesterol $(0.275, \mathrm{P}<0.05)$ were independent determinants of PWV after adjusting other possible confounding factors. The adjusted R Square for this model was 0.627 .

\section{Univariate correlation analysis in patients without inflammation}

To exclude the confounding effect of inflammation on correlation between serum albumin and PWV, univariate correlation analysis was further performed in patients without evidence of inflammation. Among the 62 patients, 30 patients with $\mathrm{CRP}$ values less than $3 \mathrm{mg} / \mathrm{L}$ were considered as noninflamed patients and selected into the subgroup analysis. The correlation analysis showed that serum albumin was still negatively correlated with PWV $(r=-0.66, \mathrm{P}<0.001$; Figure 1B). Because there were also significant correlations between serum albumin and age and diabetic status (data not shown), partial correlation analysis was performed to control the possible effect of age and diabetic status on PWV. The partial correlation showed that there was still negative correlation between serum albumin and PWV $(r=-0.412, \mathrm{P}<0.05)$. Given the relatively small sample in this non-inflamed subgroup, multiple stepwise regression analysis was not performed.

Table 2 Correlation analysis between PWV and other possible affecting factors

\begin{tabular}{lll}
\hline Variables & $\begin{array}{l}\text { Correlation } \\
\text { coefficient }\end{array}$ & P value \\
\hline Sex: female = I, male = 0 & -0.043 & NS \\
Age & 0.35 & 0.005 \\
Height & -0.036 & $\mathrm{NS}$ \\
Diabetes Mellitus: yes = I, & 0.292 & 0.021 \\
no $=0$ & & \\
Dialysis duration & 0.132 & $\mathrm{NS}$ \\
SBP & 0.493 & $<0.00 \mathrm{I}$ \\
DBP & -0.006 & $\mathrm{NS}$ \\
AHM:yes = I, no = & 0.133 & $\mathrm{NS}$ \\
Extracellular water & 0.241 & 0.077 \\
Calcium & -0.103 & $\mathrm{NS}$ \\
Phosphate & -0.244 & 0.056 \\
Total cholesterol & 0.148 & $\mathrm{NS}$ \\
CRP & 0.295 & 0.036 \\
\hline
\end{tabular}

Abbreviations: SBP, systolic blood pressure; DBP, diastolic blood pressure; AHM, antihypertensive medication; CRP, C-reactive protein. 
A

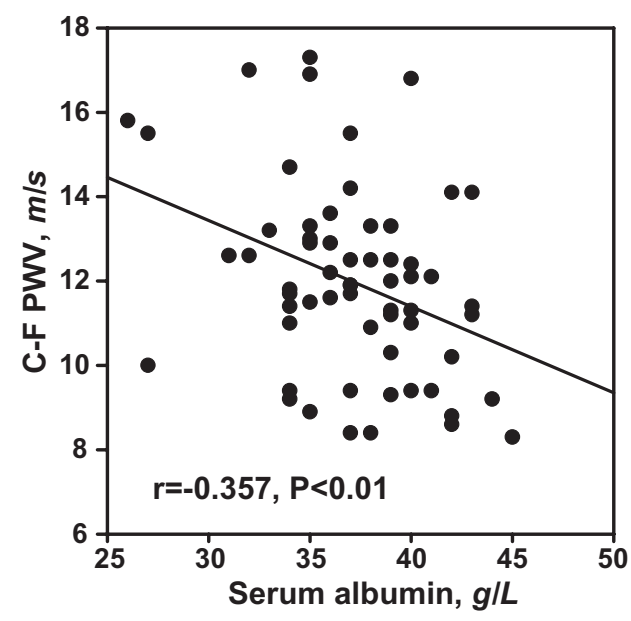

B

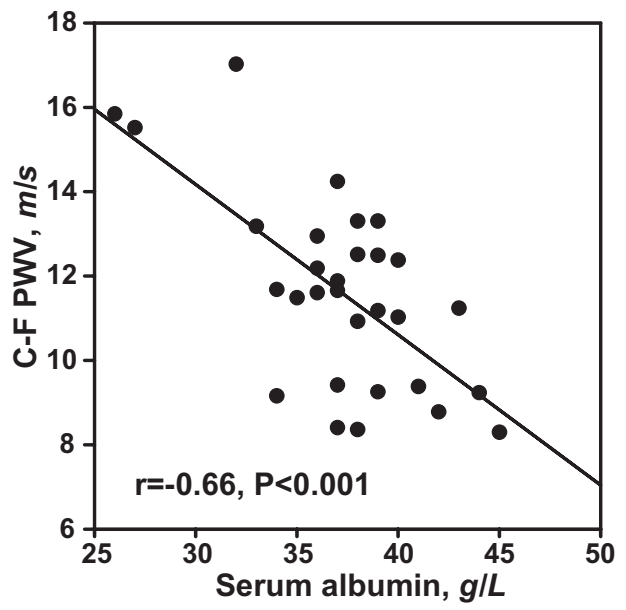

Figure I Pearson's correlation between serum albumin and carotid-femoral pulse wave velocity: $(\mathbf{A})$ In the whole study population ( $\mathrm{n}=62)$; $(\mathbf{B})$ In patients without inflammation (C-reactive protein $<3 \mathrm{mg} / \mathrm{L}$ ).

Abbreviation: C-F PWV, carotid-femoral pulse wave velocity.

\section{Discussion}

In these CAPD patients studied, there was a close association between serum albumin and PWV: 1) serum albumin was negatively correlated with PWV; 2) serum albumin was one of the independent determinants of PWV; and 3) the inverse association between serum albumin and PWV could still be observed in a subgroup of patients without evidence of inflammation.

The aortic PWV reflects central arterial stiffness (O'Rourke et al 2002). Previous studies have shown that PWV is a predictor of cardiovascular outcome in the general population (Hansen et al 2006) and in patients with hypertension (Asmar et al 2001; Laurent et al 2001; Boutouyrie et al 2002), diabetes (Cruickshank et al 2002), and hemodialysis (Blacher et al 1999) patients. On the other hand, the attenuation of PWV is related to improved clinical outcome in dialysis patients (Guerin et al 2001). Therefore, PWV was employed as the marker of aortic stiffness in the present study.

In univariate correlation analysis, we found that PWV was negatively associated with serum albumin, but positively correlated with age, diabetic status, SBP, and CRP (Table 2). While PWV failed to show any correlation with other possible affecting factors, such as sex, height, dialysis duration, DBP, antihypertensive medication, ECW, serum calcium, phosphate, total cholesterol and CRP. Given the limitation of univariate analysis in differentiating confounding factors, multiple stepwise regression analysis was further performed by including all these possible factors as independent variables. The regression analysis identified that SBP, age, serum albumin and total cholesterol were independent determinants of PWV in these dialysis patients. These four factors could explain about $62.7 \%$ of variation in PWV in principle (adjusted R Square $=0.627$, Table 3 ). It should be noted that the effect of serum albumin on PWV was independent from the mediating effect of inflammation, which was not only demonstrated in multiple regression analysis but also in the subsequent subgroup analysis. To further clarify the confounding effect of inflammation on the relationship between serum albumin and PWV, a subgroup of patients without inflammation $(\mathrm{CRP}<3 \mathrm{mg} / \mathrm{L}, \mathrm{n}=30$ ) were taken out and studied separately. The univariate correlation analysis showed that serum albumin was still negatively correlated with PWV (Figure 1B). This inverse association between serum albumin and PWV could still be observed even controlling age and diabetic status in partial correlation analysis. In line with our observation, a previous study showed that a lower albumin level was associated with a multiply adjusted risk of 2.1 for 4-year mortality compared with those with higher albumin in persons who did not have evidence of inflammation (indicated by a high interleukin- 6 level) (Reuben et al 2000).

The close association between lower serum albumin and increased PWV, the marker of increased aortic stiffness, might be explained by the following mechanisms. First, there were evidences that hypoalbuminemia was associated with increased oxidative stress in dialysis patients. Using plasma protein thiol oxidation and protein carbonyl formation as indictors of oxidative stress, Danielski and colleagues (2003) found that oxidative stress was significantly elevated 
Table 3 Multiple stepwise regression analysis for assessing the determinants of C-F PWV in CAPD patients

\begin{tabular}{llll}
\hline Model & $\boldsymbol{\beta}$ & $\mathbf{9 5 \%} \mathbf{C l}$ & P value \\
\hline SBP & 0.615 & $0.041,0.091$ & $<0.001$ \\
Age & 0.414 & $0.029,0.118$ & $<0.01$ \\
Albumin & -0.315 & $-0.315,-0.035$ & $<0.05$ \\
TC & 0.275 & $0.082,1.098$ & $<0.05$ \\
\hline
\end{tabular}

Notes: Adjusted R Square $=0.627$. Adjusted for $\operatorname{sex}($ male $=0$, female $=1)$, height, dialysis duration, diabetic status (yes $=\mathrm{I}$, no $=0$ ), diastolic blood pressure, antihypertensive medication (yes $=\mathrm{I}$, no $=0$ ), C-reactive protein, serum calcium, serum phosphate and extracellular water.

Abbreviations: SBP, systolic blood pressure;TC, total cholesterol.

in hypoalbuminemia group as compared with normoalbuminemia group. Increased oxidative stress, in turn, could accelerate atherosclerosis process. On the other hand, intravenously administered albumin was associated with increased plasma thiol levels, which improved antioxidant function (Quinlan et al 2004). Second, hypoalbuminemia was also found to have a close association with endothelial dysfunction in nephrotic syndrome (Joles et al 1999; Dogra et al 2002) and dialysis patients (Borawski et al 2001). Von Willebrand factor, an established index of endothelial dysfunction in patients with atherosclerotic cardiovascular disease (Lip and Blann 1995; Blann 2004), was found to correlate inversely with serum albumin in hemodialysis patients (Borawski et al 2001). Third, it was well known that hypoalbuminemia was often accompanied with dyslipidemia in patients with nephrotic syndrome (Cameron 1987; Joven et al 1996) and dialysis patients (Shoji et al 1991; Scolnik and Balfe 1993). Dyslimiemia played an important role in the pathogenesis of atherosclerosis and was one of the long identified risk factor for cardiovascular disease. There was also a report that in CAPD patients, albumin infusion would significantly decrease the levels of lipoprotein (a), an independent risk factor for atherosclerotic cardiovascular disease (Yang et al 1997). The aforementioned atherogenic mechanisms related to hypoalbuminemia might explain why hypoalbuminemia was identified as an independent risk factor for elevated PWV in CAPD patients.

There were some limitations in the present study. First, the cross-sectional design in this study limited our ability to infer a causal relationship between serum albumin and PWV. Indeed, totally opposite explanation might also reasonable. As a result, the relationship between serum albumin and pulse wave velocity should be verified in future interventional studies. Second, although a number of potential confounding factors such as sex, age, diabetes, lipid profile, antihypertensive medication, calcium and phosphate levels were controlled in multiple regression analysis, the existence of other unrecognized confounding variables was always possible. Third, this study was conducted in relatively older dialysis patients (mean age was 63 years), therefore, the extrapolation of the result in this study to other population, especially younger patients and nondialysis patients may not be appropriate. Indeed, there was a report that the association between hypoalbuminemia and markers of arterial stiffness could not be observed in a nondialysis population (Djousse et al 2003).

In conclusion, this study performed in dialysis patients showed that serum albumin was independently associated with PWV. The inverse association between serum albumin and PWV suggested that increased arterial stiffness might be the link between hypoalbuminemia and increased cardiovascular events and mortality in dialysis patients.

\section{Acknowledgment}

This study was supported by a grant from Cheung Kong Scholar Programme, Ministry of Education, People's Republic of China (36-1).

\section{Note}

Both Dr. Cheng and Dr. Tang are first authors of this article.

\section{References}

Asmar R, Benetos A, Topouchian J, et al. 1995. Assessment of arterial distensibility by automatic pulse wave velocity measurement: validation and clinical application study. Hypertension, 26:485-90.

Asmar R, Rudnichi A, Blacher J, et al. 2001. Pulse pressure and aortic pulse wave are markers of cardiovascular risk in hypertensive populations. Am J Hypertens, 14:91-7.

Benetos A, Rudnichi A, Safar M, et al. 1998. Pulse pressure and cardiovascular mortality in normotensive and hypertensive subjects. Hypertension, 32:560-4.

Blacher J, Guerin AP, Pannier B, et al. 1999. Impact of aortic stiffness on survival in end-stage renal disease. Circulation, 99:2434-9.

Blann AD. 2004. Von Willebrand factor and forearm blood flow as indicators of endothelial dysfunction. Atherosclerosis, 176:423-4.

Borawski J, Naumnik B, Pawlak K, et al. 2001. Endothelial dysfunction marker Von Willebrand factor antigen in haemodialysis patients: associations with pre-dialysis blood pressure and the acute phase response. Nephrol Dial Transplant, 16:1442-7.

Boutouyrie P, Tropeano AI, Asmar R, et al. 2002. Aortic stiffness is an independent predictor of primary coronary events in hypertensive patients: a longitudinal study. Hypertension, 39:2085-90.

Cameron JS. 1987. The nephrotic syndrome and its complications. Am J Kidney Dis, 10:157-71.

Cheng LT, Tang W, Wang T. 2005. Strong association between volume status and nutritional status in peritoneal dialysis patients. Am J Kidney Dis, 45:891-902.

Cooper BA, Penne EL, Bartlett LH, et al. 2004. Protein malnutrition and hypoalbuminemia as predictors of vascular events and mortality in ESRD. Am J Kidney Dis, 43:61-6.

Cruickshank K, Riste L, Anderson SG, et al. 2002. Aortic pulse-wave velocity and its relationship to mortality in diabetes and glucose intolerance: An integrated index of vascular function? Circulation, 106:2085-90. 
Danielski M, Ikizler TA, McMonagle E, et al. 2003. Linkage of hypoalbuminemia, inflammation, and oxidative stress in patients receiving maintenance hemodialysis therapy. Am J Kidney Dis, 42:286-94.

Dart AM, Kingwell BA. 2001. Pulse pressure - a review of mechanisms and clinical relevance. J Am Coll Cardiol, 37:975-84.

Djousse L, Rothman KJ, Cupples A, et al. 2003. Relation between serum albumin and carotid atherosclerosis The NHLBI Family Heart Study. Stroke, 34:53-7.

Dogra GK, Herrmann S, Irish AB, et al. 2002. Insulin resistance, dyslipidaemia, inflammation and endothelial function in nephrotic syndrome. Nephrol Dial Transplant, 17:2220-5.

Enia G, Mallamaci F, Benedetto FA, et al. 2001. Long-term CAPD patients are volume expanded and display more severe left ventricular hypertrophy than haemodialysis patients. Nephrol Dial Transplant, 16:1459-64.

Foley RN, Parfrey PS, Harnett JD, et al. 1995. Clinical and echocardiographic disease in patients starting end-stage renal disease therapy. Kidney Int, 47:186-92.

Foley RN, Parfrey PS, Harnett JD, et al. 1996. Hypoalbuminemia, cardiac morbidity, and mortality in end-stage renal disease. J Am Soc Nephrol, 7:728-36.

Guerin A, Blacher J, Pannier B, et al. 2001. Impact of aortic stiffness attenuation on survival of patients in end-stage renal disease. Circulation, 103:987-92.

Hansen TW, Staessen JA, Torp-Pedersen C, et al. 2006. Prognostic value of aortic pulse wave velocity as index of arterial stiffness in the general population. Circulation, 113:664-70.

Joles JA, Stroes ES, Rabelink TJ. 1999. Endothelial function in proteinuric renal disease. Kidney Int Suppl, 71:S57-S61.

Joven J, Espinel E, Simo JM, et al. 1996. The influence of hypoalbuminemia in the generation of nephrotic hyperlipidemia. Atherosclerosis, 126:243-52.

Kalantar-Zadeh K, Kilpatrick RD, Kuwae N, et al. 2005. Revisiting mortality predictability of serum albumin in the dialysis population: time dependency, longitudinal changes and population-attributable fraction. Nephrol Dial Transplant, 20:1880-8.
Laurent S, Boutouyrie P, Asmar R, et al. 2001. Aortic stiffness is an independent risk predictor of all-cause and mortality in hypertensive patients. Hypertension, 37:1236-41.

Lip GY, Blann AD. 1995. Von Willebrand factor and its relevance to cardiovascular disorders. Br Heart J, 74:580-3.

O'Rourke MF, Staessen JA, Vlachojannis JA, et al. 2002. Clinical application of arterial stiffness:definitions and reference values. $\mathrm{Am} J$ Hypertens, 15:426-44.

Quinlan GJ, Mumby S, Martin GS, et al. 2004. Albumin influences total plasma antioxidant capacity favorably in patients with acute lung injury. Crit Care Med, 32:755-9.

Reuben DB, Ferrucci L, Wallace R, et al. 2000. The prognostic value of serum albumin in healthy older persons with low and high serum interleukin-6 (IL-6) Levels. J Am Geriatr Soc, 48:1404-7.

Scolnik D, Balfe JW. 1993. Initial hypoalbuminemia and hyperlipidemia persist during chronic peritoneal dialysis in children. Perit Dial Int, 13:136-9.

Shoji T, Nishizawa Y, Nishitani H, et al. 1991. Roles of hypoalbuminemia and lipoprotein lipase on hyperlipoproteinemia in continuous ambulatory peritoneal dialysis. Metabolism, 40:1002-8.

Van Bortel LM, Struijker-Boudier HA, Safar ME. 2001. Pulse pressure, arterial stiffness, and drug treatment of hypertension. Hypertension, 38:914-21.

Vlachopoulos C, O’Rourke M. 2000. Diastolic pressure, systolic pressure, or pulse pressure? Curr Hypertens Rep, 2:271-9.

Wong CS, Hingorani S, Gillen DL, et al. 2002. Hypoalbuminemia and risk of death in pediatric patients with end-stage renal disease. Kidney Int, 61:630-7.

Yang WS, Min WK, Park JS, et al. 1997. Effect of increasing serum albumin on serum lipoprotein(a) concentration in patients receiving CAPD. Am J Kidney Dis, 30:507-13. 